\title{
REPERTOIRE
}

\section{DES ESPÈCES ET DES GENRES NOUVEAUX ${ }^{(1)}$}

\section{Protophytes}

Eccrinopsis mercieri R. Poisson. Eccrinide. Rectum. Oniscus asellus. France, Normandie, C. R. Acad. des sc., CLXXXVI, 1928, p. 1765.

M. LANGERON.

\section{Myxophycées}

Anabaeniolum Iangeroni G. Nadson et N. Krassilnikov. Oscillariaceæ. Cæcum. Cavia cobaya. Russie. C. R. Acad. des sc., CLXXXVII, 1928, p. 176.

M. L.

\section{Hyphomycètes}

Aspergillus dessyi C. Spegazzini. Conidiosporaceæ. Peau. Homme. République Argentine. Physis, VIII, 1925, p. 115.

Sterigmatocystis tropicalis da Matta. Conidiosporacer. Pied. Homme. Brésil. Bol. Inst. brasileiro de sc., III, 1928, p. 51.

M. L.

\section{Ascomycètes}

Stigmatomyces ephydrae L. Mercier et R. Poisson. Laboulbeniaceæ. Tégument. Ephydra riparia (Diptère). Colleville-sur-Orne (Calvados). Bull. Soc. zool. de France, LII, 1927, p. 226.

M. L.

\section{Rhizopodes}

Councilmania dissimilis Kofoid. Amœebidæ. Intestin. Homme. Amérique du Nord. Univ. California public. zool., XXXI, 1927, p. 7.

M. L.

(1) La Direction des Annales de Parasitologie prie instamment les auteurs qui décrivent des espèces parasitaires nouvelles de vouloir bien lui adresser leurs travaux, 15, rue de l'Ecole-de-Médecine, ḋ Paris, afin qu'il en soit tenu compte dans le plus court délai. A défaut de tirés à part, on peut envoyer une liste des espèces nouvellement décrites, avec indications bibliographiques.

Annales de Parasitologie, $\mathrm{T}$. VII, $\mathrm{x}^{\circ} 1 .-1^{\mathrm{er}}$ janvier 1929, p. 91-96. 


\section{Sporozoaires}

Eimeria mephitIdis J. Andrews. Eimeridæ. Intestin. Mephilis (skauk de l'Amérique du Nord). Amérique du Nord. Journ. of paras., XIV, 1928. p. 193 .

Monocystis mrazeki J. Hahn. Monocystida. Intestin. Rhynchelmis limosella, $R$. komareki Hrabé. Bohême, Moravie, Balkans. Arch. für Protist., LXII, 1928, p. 1.

Françaiella colchica Yakimoff. Piroplasmidæ. Sang. Bovidés. Nord du Caucase. Arch. für Prolist., LXII, 1928, p. 105.

Plasmodium joyeuxi M. Léger. Plasmodidæ. Sang. Cercopilhecus callitrichus. Sénégal et Guinée. Ann. Inst. Pasteur Paris, XLII, p. 780.

Myxobolus noguchii C. Pinto. Myxosporidie. Branchies. Serrasalmo spilopleura Kner. Rio Turvo, Pirangy, Etat de St-Paul, Brésil. Boletim biologico, no 12, 1928, p. 42.

Myxobolus stokesi C. Pinto. Myxosporidie. Tumeur sous-cutanée de la tête. Jeune Pimelodella (?) (Poisson). Rio Turvo, Pirangy, Etat de St-Paul, Brésil. Boletim biologico, no 12,1928, p. 42.

Henneguya iheringi C. Pinto. Myxosporidie. Branchies. Serrasalmo spilopleura Kner. Rio Turvo, Pirangy, Etat de St-Paul, Brésil. Boletim biologico, no 12,1928 , p. 43.

Haemoproteus de melloi Venancio da Silva. Hæmoproteidæ. Sang. Plernistes lucani (perdrix de l'Angola). Rev. med. de Angola, no 5, 1927, p. 99.

M. L.

\section{Flagellés}

Trypanosoma morai Venancio da Silva. Trypanosomidr. Sang. Bubulcus ibis (Héron). Ambriz, Angola. Rev. med. de Angola, no 5, 1927, p. 97.

Pseudotrichonympha belari $\mathrm{F}$. de Mello. Trichonymphida. Intestin. Leuco. termes indicola. Nova Goa. Nom. nov. pro Trichonympha agilis de Mello, 1918, nec Leidy, Holomastigotoides herlwigi Andrade et Guimaraes, 1922, nec Grassi. Arquivos Escola med.-cir. Nova Goa, ser. A, fasc. 1, 1927, p. 22.

Holomastigotoides annandalei F. de Mello. Trichonymphidæ. Intestin. Leucolermes indicola. Nova Goa. Syn. : Leidya annandalei F. de Mello, 1918 pro parle. Arquivos Escola med.-cir. Nova Goa, ser. A, fasc. 3, 1928, p. 249.

Holomastigotoides koidzumii F. de Mello. Trichonymphidre. Intestin. Lencolermes indicola. Nova Goa. Arquivos Escola med.-cir. Nova Goa, ser. A, fasc. 3,1928 , p. 250.

Holomastigotoides metchnikovi F. de Mello. Trichonymphidr. Intestin. Leucotermes indicola. Nova Goa. Syn. : Leydia metchnikovi F. de Mello, 1918 pro parle nec L. metchnikovi França, 1916), Pirsonympha grassii F. de Mello, 1928. Arquivos Escola med.-cir. Nova Goa, ser. A, fasc. 3, 1928, p. 251.

Holomastigotoides kempi F. de Mello. Trichonymphidæ. Intestin. Leucolermes indicola. Nova Goa. Syn.: Leydia kempi F. de Mello, 1918 pro parte. Arquivos Escola med.-cir. Nova Goa, ser. A, fasc. 3, 1928, p. 251.

Holomastigotoides gigas F. de Mello. Trichonymphidæ. Intestin. Leucolermes indicola. Nova Goa. Syn.: Leydia metchnikovi F. de Mello, 1918 pro 
parle, nec França, 1916. Arquivos Escola med.-cir. Nova Goa, ser. A, fasc. 3, 1928, p. 251.

Spirotrichonympha rotunda F. de Mello. Trichonymphidæ. Intestin. Leucotermes indicola. Nova Goa. Arquivos Escola med.-cir. Nova Goa, ser. A, fasc. 3,1928 , p. 261.

M. L.

\section{Infusoires}

Cepedea brumpti E.-H. Cordero. Opalinidx. Intestin. Hyla raddiana. Uruguay. 4a Reunion. Soc. argentina patol. region. Norte, Santiago del Estero, 1928. Bol. Inst. clin. quir., Buenos-Aires, IV, 1928, p. 589.

Nyctotherus ampullariarum E.-H. Cordero. Heterotrichida. Intestin. Ampullaria canaliculala Lam., A. insularium d'Orb., A. megasloma Sow. Uruguay. ha Reunion. Soc. argentina patol. region, Norte, Santiago del Eslero, 1928. Bol. Inst. clin. quir., Buenos-Aires, IV, 1928, p. 591.

M. L.

\section{Cestodes}

Raillietina (Raillietina) boueti Joyeux et Baer. Davaineidæ. Intestin. Francolinus bicalcaratus (Galliformes). Agovagon (Dahomey). Monographie II. Soc. de pathol. exol., 1928, p. 30.

Raillietina (Fuhrmanetta) bucerotidarum Joyeux et Baer. Davaineida. Intestin. Melanobucco rquatorialis (Coraciiformes). Labé (Guinée française). Monographie II. Soc. de pathol. exol., 1928, p. 33.

Deltokeras campylometra Joyeux et Baer. Dilepidida. Intestin. Pyromelana franciscana et Pealhetriopsis macrura (Passériformes). Abomey (Dahomey). Monographie II. Soc. de palhol. exot., 1928, p. 38.

Paruterina africana Joyeux et Baer. Dilepididx. Intestin. Potamorhynchus senegalus (Passériformes). Bohicon (Dahomey). Monographie II. Soc. de pathol. exol., 1928, p. 41.

Paruterina guineensis Joyeux et Baer. Dilepidida. Intestin. Coccystes cafer (Coccygiformes). Abomey (Dahomey). Monographie II. Soc. de palhol. exol., 1928 , p. 43.

Oligorchis toxometra Joyeux et Baer. Hymenolepididx. Intestin. Gallinago sp. (Charadriiformes). Labé (Guinée française). Monographie II. Soc. de salhol. exot.. 1928, p. 46.

Hymenolepis maclaudi Joyeux et Baer. Hymenolepidida. Intestin. Crocidura slampflii (Insectivores). Abomey (Dahomey). Monographie II. Soc. de pathol. exol., 1928, p. 49.

Hymenolepis (Echinocotyle) dolosa Joyeux et Baer. Hymenolepididx. Intes-

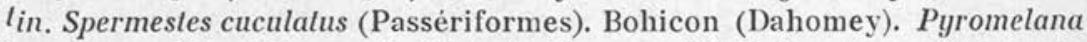
franciscana (Passériformes). Sénégal. Monographie II. Soc. de palhol. exot., 1928, p. 49.

Ch. Joyeux.

Bouchardia J. Guiart. Bouchardida nov. fam. Espèce týpe : B. crassiceps (Dies. 1850). Assoc. française avancement des sc., Lyon, 1926, 1927, p. 398. 
Rufferia J. Guiart. Rufferidæ nov. fam. Espèce type : R. tubiceps (Leuckart, 1819). Assoc. francaise avancement des sc., Lyon, 1926, 1927, p. 398.

Pierretia J. Guiart. Rufferidæ. Espèce type : P. carchariæ (von Linstow, 1878). Assoc. française avancemenl des sc., Lyon, 1926, 1927, p. 398.

Vaullegeardia J. Guiart. Vaullegeardidæ nov. fam. Espèce type: V. moniezi (Railliet, 1899). Assoc. française avancement des sc., Lyon, 1926, 1927, p. 399.

Grillotia J. Guiart. Lacistorhynchidæ nov. fam. Espèce type : G. erinaceus (van Beneden, 1858). Assoc. francaise avancement des sc., Lyon, 1926, 1927, p. 399 .

Armandia J. Guiart. Eutetrarhynchidæ nov. fam. Espèce type : A. minula (van Beneden, 1849). Assoc. française avancement des sc., Lyon, 1926, 1927, p. 401.

Gilquinia J. Guiart. Bouchardidæ nov. fam. Espèce type : G. tetrabothria (van Beneden, 1849). Assoc. française avancement des sc., Lyon, 1926. 1927, p. 401.

R.-Ph. Dollfus.

\section{Trématodes}

Acanthotrema Travassos. Haplorchidr. Espèce type : A. acantholrema. C. R. Soc. biol., XCIX, 1928 , p. 884 .

Acanthotrema acanthotrema Travassos. Aplorchide. Intestin. Sterna maxima (Lariformes). Rio de Janeiro. C. R. Soc. biol., XCIX, 1928, p. 884.

Schistosoma faradjei Walkiers. (1) Schislosomatidæ. Intestin? Homme. Haut Ouellé. Ann. Soc. belge de méd. trop., VIII, 1928, p. 21.

Opisthodiscus americanus Holl. Diplodiscinæ. Colon. Triturus viridescens (Sauriens). Durham. Journ. of helminthology, VI, 1928, p. 175.

Gorgoderina intermedia Holl. Gorgoderida. Vessie urinaire. Triturus viridescens (Sauriens). Lakeview (Caroline du nord). Journ. of helminthology, VI, 1928, p. 178.

Brachycœlium trituri Holl. Brachycoline. Intestin. Trilurus viridescens (Sauriens). Lakeview (Caroline du nord). Journ. of helminthology, VI, 1928, p. 180.

Ch. Joyeux.

Opisthogonimus megabothrium C. Pereira. Lepodermalida. Wisophage et bouche. Rhadinoea merremii, Ophis merremii (Ophidiens). Etat de St-Paul, Brésil. Bolelim biologico, no 12, 1928, p. 50.

Megalogonia ictaluri E.-W. Surber. Allocreadide (?). Inteslin. Ictalurus punclalus (Poisson). St. Croix River, Stillwater, Minnesota. Journ. of parasilology, XIV, 1928, p. 269.

M. LANGERON.

(1) L'auteur crée cette nouvelle espèce sous toutes réserves, l'adulte n'ayant pas été observê, et le diagnostic étant seulement fondé sur l'absence d'éperon des œufs émis dans les selles, chez 5 indigènes souffrant de diarrhée sanglante. 


\section{Nématodes}

Hystrignathus politus P. Artigas. Rhabditide. Diverticules de l'intestin. Passaloïdes (Coléoptères lamellicornes). Bofete, Etat de St-Paul, Brésil. Boletim biologico, no 12,1928 , p. 71 .

Lepidonema tarda P. Artigas. Rhabditide. Diverticules de l'intestin. Passaloïdes (Coléoptères lamellicornes). Bofete, Etat de St-Paul, Brésil. Boletim biologico, no 12,1928, p. 72.

Hepaticola anthropopitheci J. Troisier, R. Deschiens, H. Limousin et M. Delorme. Trichinellidx. CEufs dans le foie. Anlhropopithecus troglodytes. L. Kindia, Afrique occidentale française. Ann. Inst. Pasteur Paris, XLII, 1928 , p. 834 .

Tetrameres paucispina J.-H. Sandground. Spiruridx. Proventricule. Amblyramphus holosericeus. (Oiseau sud-américain mort au Franklin Park zoological Garden de Boston). Journ. of parasilology, XIV, 1928, p. 268.

Spiroxys torquata J.-N. Karve. Gnathostomidz. Estomac. Emida granosa intermedius. Nagpur, C. P., Inde anglaise. Ann. of trop. med. and parasitology, XXII, 1918, p. 268.

Filaria patersoni S. Mazza Filaridæ. Cavité péritonéale. Holochilus vulpinus Brandt (Rongeur). San Martin del Tabacal, Salta (République Argentine). 4 a Reunion, Soc. argentina patol. region. Norte, Santiago del Estero, 1928. Bol. Inst. clin. quir. Buenos-Aires, IV, 1928, p. 632.

Microfilaria parodii S. Mazza et I. Franke. Filaridæ. Sang. Cyanocorax chrysops (Viellot). Nord de la République Argentine. 4a Reunion, Soc. argenlina. patol. region. Norle, Santiago del Eslero, 1928, Bol. Inst. clin. quir. Buenos-Aires, IV, 1928, p. 626.

Microfilaria corderoi S. Mazza et I. Franke. Filarida. Sang. Scapanus lencopogon (Valenciennes). Nord de la République Argentine. 4a Reunion, Soc. argentina palol. region. Norle, Santiago del Estero, 1928. Bol. clin. quir. Buenos-Aires, IV, 1928, p. 627.

Microfilaria fonsecai S. Mazza et I. Franke. Filaridæ. Sang. Coryphospingus cucullatus (P.-L. Müll.). Nord de la République Argentine. 4a Reunion, Soc. argentina patol. region, Norte, Sanliago del Eslero, 1928. Bol. clin. quir., Buenos-Aires, IV, 1928, p. 627.

M. L.

Physaloptera joyeuxi Ch. Joyeux, E. Gendre, J.-G. Baer. Acuariidw. Intestin. Phacochœrus africanus. Goungoun (Dahomey). Monographie II. Soc. path. exol., 1928, p. 71.

Aphelenchus retusus N.-A. Cobb. Anguillulinida. Pupe morte. Chretopsis anea. Milford (Iowa). Helminthol. Soc. of Washinglon. Journal of parasitology, XIV, 1927, p. 60.

Acanthocéphales

J. Sautet.

Quadrigyrus cholodovskyi N.-N. Kostylew. Ncoechinorhynchidx. Intestin. Varicorinus sevangi (Filippi). Lac Goktscha, Arménie. Clrlbl. für Bakl., Orig., CVIII, 1928, p. 147. 
Echinorhynchus baeri N.-N. Kostylew. Intestin. Salmo ischan Kessler. Lac Goktscha, Arménie. Ctrlbl. für Bakt., Orig, .CVIII, 1928, p. 148.

M. LANGERON.

\section{Acariens}

Ornithodoros foleyi Parrot. Ixodidx. Hoggar, confluent de l'oued Tinikert et de l'Igharghar. Bull. Soc. palhol. exot., XXI, 1928, p. 520.

M. L.

Trombicula pseudo schüffneri Walch. Trombididx. Sumatra, Overged. nit hel Gen. Tijd. voor Ned. Indï̈, VI, 1927, p. 924.

Trombidium glabrum Walch. Trombididx. Sumatra. Overged. uit het Geneesk. Tidj, voor Neder. Indië, VI, 1927, p. 926.

Trombidium globulare Walch. Trombididx. Célèbes. Overged. uil het Geneesk. Tidj. voor Neder. Indië, VI, 1927, p. 929.

H. Galliard.

\section{Rectification de Nomenclature}

Nous recevons du Docteur Maurice-C. Hall, chef de la division zoologique du Bureau de l'Industrie animale à Washington, la communication suivante, au sujet de la rectification de nomenclature proposée par R.-Ph. Dollfus dans ces Annales (VI, $\mathrm{n}^{\circ} 4,1^{\text {er }}$ octobre 1928, p. 487).

«. Dans le numéro d'octobre 1928 des Annales de Parasitologie, j’ai remarqué qu'à la page 487 une note de Dollfus propose le nom de Steineriella pour remplacer Steineria, Travassos, nec Filipjev, 1922.

«Le fait que Travassos a proposé un homonyme est venu à notre connaissance l'an dernier en notant son travail et le $\mathrm{D}^{\mathrm{r}}$ Hassal lui a écrit, le 30 juin 1927, en l'engageant à proposer un autre nom.

« C'est l'habitude de notre laboratoire, d'accord avec les Recommandations de la Commission Internationale de Nomenclature zoologique et avec le code d'éthique de la Société d'helminthologie. Tous deux prescrivent que toute personne remarquant un homonyme doit écrire à l'auteur et l'engager à proposer un nouveau nom : ce nouveau nom doit être proposé dans l'année. Dans le cas particulier, Travassos a déjà proposé un nouveau nom, ce qui fait que Steineriella tombe en synonymie. »

M.-C. HaLl.

Le Gérant: F. Amirault. 